\title{
Experimental Organism Benign Keratoacanthoma
}

National Cancer Institute

\section{Source}

National Cancer Institute. Experimental Organism Benign Keratoacanthoma. NCI

Thesaurus. Code C117977.

A benign neoplasm in the superficial dermis with direct association to the epidermis, composed of well differentiated squamous epithelium and a central cavity filled with concentric layers of keratin; a pore (opening in the epidermis) may be present. (CDISC) 